\title{
On the nature of optical nuclei in FR I radio-galaxies from ACS/HST imaging polarimetry ${ }^{\star}$
}

\author{
A. Capetti ${ }^{1}$, D. J. Axon ${ }^{2}$, M. Chiaberge ${ }^{3,6}$, W. B. Sparks ${ }^{3}$, F. Duccio Macchetto ${ }^{3,4}$, M. Cracraft ${ }^{3}$, and A. Celotti ${ }^{5}$ \\ 1 INAF - Osservatorio Astronomico di Torino, Strada Osservatorio 20, 10025 Pino Torinese, Italy \\ e-mail: capetti@to.astro.it \\ 2 Department of Physics, Rochester Institute of Technology, 85 Lomb Memorial Drive, Rochester, NY 14623, USA \\ 3 Space Telescope Science Institute, 3700 San Martin Drive, Baltimore, MD 21218, USA \\ 4 Affiliated with ESA's Research \& Space Science Department \\ 5 SISSA/ISAS, via Beirut 2-4, 34014 Trieste, Italy \\ 6 INAF - Istituto di Radioastronomia, via Gobetti 101, 40129 Bologna, Italy
}

Received 28 February 2007 / Accepted 7 June 2007

\section{ABSTRACT}

\begin{abstract}
We obtained optical imaging polarimetry with the ACS/HRC aboard the HST of the 9 closest radio-galaxies in the 3C catalogue with an FR I morphology. The nuclear sources seen in direct HST images in these galaxies are found to be highly polarized with levels in the range $\sim 2-11 \%$ with a median value of $7 \%$. We discuss the different mechanisms that produce polarized emission and conclude that the only viable interpretation is a synchrotron origin for the optical nuclei. This idea is strengthened by the analogy with the polarization properties of BL Lac objects, providing also further support to the FR I/BL Lac unified model. This confirms previous suggestions that the dominant emission mechanism in low luminosity radio-loud AGN is related to non-thermal radiation produced by the base of their jets.

In addition to the nuclear polarization (and to the large scale optical jets), polarization is detected co-spatially with the dusty circumnuclear disks, likely due to dichroic transmission; the polarization vectors are tangential to the disks as expected when the magnetic field responsible for the grains alignment is stretched by differential rotation.

We explored the possibility to detect the polarimetric signature of a misaligned radiation beam in FR I, expected in our sources in the frame of the FR I/ BL Lac unification. We did not find this effect in any of the galaxies, but our the results are not conclusive on whether a misaligned beam is indeed present in FR I.
\end{abstract}

Key words. galaxies: active - galaxies: elliptical and lenticular, cD - galaxies: nuclei - galaxies: jets - polarization

\section{Introduction}

The presence of a radio-source represents a common manifestation of nuclear activity associated with elliptical galaxies, in particular for the brightest members of this class. For example, among galaxies brighter than $M_{B}<-21$, more than $20 \%$ are radio-active with luminosities $L_{408 \mathrm{MHz}}>10^{23.5} \mathrm{~W} \mathrm{~Hz}^{-1}$ (Colla et al. 1975). Most of them are low luminosity radio-galaxies (LLRG) and show the characteristic edge-darkened FR I radio morphology (Fanaroff \& Riley 1974).

HST imaging of low luminosity radio-galaxies proved to be a very powerful tool for a better understanding of the properties of this class of Active Galactic Nuclei (AGN) as it allowed us to isolate, for the first time, their genuine optical nuclear emission from that of the host galaxies. Analysis of $R$ band HST images of the sample formed by all the 33 FR I sources belonging to the $3 \mathrm{C}$ catalogue showed that unresolved optical nuclear sources are detected in the great majority of these galaxies (Chiaberge et al. 1999). Their optical and radio nuclear luminosities show a clear correlation, suggestive of a common non-thermal synchrotron origin, most likely from the base of their relativistic jets.

^ Based on observations obtained at the Space Telescope Science Institute, which is operated by the Association of Universities for Research in Astronomy, Incorporated, under NASA contract NAS 526555 .
Recently we found a similarly tight connection between the radio and X-ray luminosities measured from Chandra observations (Balmaverde et al. 2006), confirming the trends already suggested by lower resolution X-ray telescopes (e.g. Capetti et al. 2000b; Hardcastle \& Worrall 2000; Trussoni et al. 2003; Hardcastle et al. 2003). Furthermore, the correlation between radio, optical and X-ray emission extends to the large population of radio-loud nuclei found in early-type galaxies, with luminosities smaller by a factor as large as 1000 with respect to classical low-luminosity radio-galaxies (Balmaverde \& Capetti 2006).

The picture which emerges is that the nuclear emission in low luminosity radio-loud AGN is dominated by non-thermal emission from the base of their jets.

This result also provides strong support for the identification of FR I radio-galaxies as the misoriented population associated with BL Lac objects. Indeed, in the framework of this unifying scheme, the non-thermal radiation from the jet, which dominates the optical emission in BL Lacs, is expected to be present also in FR I radio-galaxies, although de-amplified.

Alternative explanations for the origin of the nuclear sources (e.g. stellar nuclear cusps, emission from radiatively efficient accretion disks) appear to be less likely, principally as none of them naturally accounts for the tight correlation between the radio, optical and X-ray luminosities. Apparently, the only viable alternative are radiatively inefficient accretion flows (RIAF, Narayan \& Yi 1995). Indeed, the measured nuclear optical luminosities 
translate for a $10^{9} M_{\odot}$ black hole as typical of FR I sources, into a fraction as small as $\lesssim 10^{-5}-10^{-7}$ of the Eddington luminosity. This indicates that accretion occurs at low rate and/or in a low radiative regime.

Polarimetric observations, particularly those at radio and optical wavelengths, played an important role in extragalactic astrophysics. From the discovery of synchrotron radiation to the first good evidence for AGN unification, to the discovery of variable linear polarization in the absorption troughs of BAL QSO, polarization studies often provide the best and sometimes the only clues we have regarding the morphology and the radiation processes in these diverse sources. This is also clearly the case for understanding the origin of the nuclear emission in radio-galaxies.

In fact, a simple and direct test of the scenario presented above can be performed by measuring the nuclear polarization of FR I radio-galaxies. If their nuclear emission is dominated by synchrotron radiation we expect to detect significant polarization.

Ground based measurements (see e.g. Impey et al. 1991) have been hampered by the dominant dilution of unpolarized starlight as their nuclear sources only account for a few per cent of the total flux in typical ground based apertures. Thanks to the high resolution of HST it is possible to isolate the nuclear emission from that of the host galaxy and to measure its intrinsic polarization.

Previously we obtained polarization measurements with HST for two radio-galaxies. For the first galaxy, M 87 (AKA 3C 274) the nucleus was found to be only marginally polarized $(P=1.6 \pm 0.7 \%)$ in the optical (Capetti et al. 1997). Conversely, the nucleus of the second radio-galaxy, Centaurus A, is highly polarized, with $P=11.1 \pm 0.2 \%$ at $2 \mu \mathrm{m}$ (Capetti et al. 2000a). This high polarization can be interpreted in two different scenarii. The first possibility is that we are seeing the infrared counterpart of the synchrotron radio-core and this accounts naturally for its polarization. However, since the orientation of the nuclear polarization $(\mathrm{PA}=148.2 \pm 1.0)$ is almost exactly perpendicular to the radio jet $(\mathrm{PA}=55)$, the polarized component might also be a very compact scattering region (with radius smaller than $1 \mathrm{pc}$ ) and in this case the nucleus is hidden to our view. We are thus left with a substantial ambiguity in interpreting the observed nuclear polarization based on observations of a single object.

Only with measurements of the nuclear polarization for a complete sample of objects it will be possible to settle this issue. It is also essential that the sample is unbiased with respect to orientation, so that the objects can be considered to have their jet axes randomly distributed with respect to the line of sight. The $3 \mathrm{C}$ sample is selected at low radio frequency where the extended emission, which does not depend on orientation, dominates over the nucleus; thus the flux threshold of the catalogue selection criterion does not introduce any orientation bias. We then selected, from the complete sample of the 33 FR I part of the 3C sample, the closest sources setting a redshift threshold at $z<0.025$. This lead to the final sample of 9 sources. In all of them an optical nuclear source has been detected by Chiaberge et al. (1999).

The paper is organized as follows: in Sect. 2 we describe the observations of the 9 science targets. The results of Appendix A, where we discuss the capabilities of the HRC as a polarimeter, are used in Sect. 3 to measure the polarization of the optical nuclei, while in Sect. 4 we describe and discuss the nature of regions of extended polarization. The origin of the nuclear emission is explored in Sect. 5. Finally in Sect. 6 we discuss our results and present our conclusions.
Table 1. Observations log.

\begin{tabular}{lcccc}
\hline \hline Name & \multicolumn{1}{c}{ Filter } & Exp. time & Date & PAV3 \\
\hline 3C 31 & F606W + POLiV & 796 & $02-08-25$ & 50.6 \\
3C 66B & $F 606 W+$ POLiV & 824 & $02-08-21$ & 64.1 \\
3C 264 & F606W + POLiV & 300 & $02-12-05$ & 115.9 \\
3C 270 & $F 606 W+$ POLiV & 782 & $02-07-07$ & 291.4 \\
3C 272.1 & $F 606 W+$ POLiV & 782 & $02-12-17$ & 115.2 \\
3C 274 & F606W + POLiV & 300 & $02-12-10$ & 115.6 \\
3C 296 & F606W + POLiV & 782 & $02-07-10$ & 296.8 \\
3C 465 & F606W + POLiV & 786 & $02-09-06$ & 48.4 \\
3C 449 & F606W + POLiV & 796 & $02-07-05$ & 17.0 \\
\hline
\end{tabular}

\section{Observations and data reduction}

Images of the 9 radio-galaxies were obtained with the High Resolution Camera (HRC) of the Advanced Camera for Survey (ACS). Its pixel size is 0 ' $^{\prime} 028 \times 00^{\prime} 025$, for a nominal $29^{\prime \prime} \times$ $26^{\prime \prime}$ field of view. The broad-band filter F606W (centered at $5900 \AA$ and with a width of $2300 \AA$ ) was used in combination with the three visible polarizers, POL0V, POL60V and POL120V. The polarizers have their principal planes oriented nominally at PA 0,60 and 120 degrees.

Two exposures of equal length have been obtained for each filter combination to enable removal of cosmic ray events. The total exposures times per polarizer range from 782 to $824 \mathrm{~s}$ for most objects, while they are $300 \mathrm{~s}$ for two sources (namely 3C 264 and 3C 274). See Table 1 for the observations log.

The individual images were processed through the standard HST pipeline and the exposures were combined to remove cosmic ray events with the multidrizzle task in the STSDAS dither package.

The images obtained through the three polarizers can be combined to derive the Stokes parameters $I, Q$ and $U$ using the following relationships

$$
\begin{aligned}
& I=\frac{2}{3}\left(I_{0}+I_{60}+I_{120}\right) \\
& Q=\frac{2}{3}\left(2 \cdot I_{0}-I_{60}-I_{120}\right) \\
& U=\frac{2}{\sqrt{3}}\left(I_{60}-I_{120}\right)
\end{aligned}
$$

where $I_{0}, I_{60}$ and $I_{120}$ are the count rates measured with the three polarizers. The Stokes parameters can be then used to measure the polarized flux $I_{P}=\left(Q^{2}+U^{2}\right)^{1 / 2}$, the percentage of linear polarization $P=\frac{I_{P}}{I}$ and the polarization position angle (in the instrument reference frame) $\theta_{\text {instr. }}=0.5 \cdot \tan ^{-1}\left(\frac{U}{Q}\right)$. The polarization PA can be referred to the sky reference frame, following Biretta et al. (2004); Biretta \& Kozhurina-Platais (2004), as $\theta=\theta_{\text {instr. }}-69.6+$ PAV3, where PAV3 is the orientation of the telescope V3 axis during the observations.

The statistical errors in the Stokes parameters were computed assuming Poisson noise in the images. The errors on the degree and position angle of the polarization are then estimated as

$\sigma_{P}=\sqrt{2} I_{i}^{-1 / 2}$ and $\sigma_{\theta}=\sqrt{2} I_{i}^{-1 / 2} P^{-1}$

respectively, where $I_{i}$ are the total counts in one of the polarizers. As explained in Appendix B we preferred not to correct for the polarization bias. 
In Appendix A we discuss the capabilities of the HRC as a polarimeter, analyzing the complete set of observations of standard targets available from the calibration program and assessing the accuracy level of polarization measurements with this instrument. Here we briefly summarize the main results of this analysis and refer for the details to this Appendix.

Measurements of the count rates through each of the 3 polarizers for two unpolarized standard stars indicate a substantial departure from an ideal polarimeter, for which one would expect equal count rates. We investigated the origin of this effect by using also observations of a polarized star, observed in 5 different epochs and at different telescope orientations. Our conclusion is that the expected polarization for all calibration targets can be accurately reproduced if there is a difference in the transmission of the 3 polarizers, with POL0V being about $10 \%$ less efficient than POL60V and POL120V. By applying the appropriate correction to the count rates we reproduced accurately the expected values for the standard stars to within $0.4 \%$. This value can be considered as upper limit to the uncertainties due to any residual instrumental polarization, as it also includes errors due to the flat field accuracy on the polarization measurements and to the statistical uncertainties.

This analysis was performed using relatively large apertures (50 pixels) while for our science targets we are interested in measuring the nuclear polarization on smaller apertures. On a scale of a few pixels, there might be e.g. differences in the PSF of each polarizer, leading spurious polarization measurements. We investigated this issue integrating the count rates through several synthetic apertures and concluded that robust polarimetric measurements can be obtained with apertures of radius as small as $r \gtrsim 3$ pixels, with no appreciable differences in accuracy.

In the analysis of our science targets we therefore first applied the appropriate correction to the count rates (dividing by 1.095 and 1.109 the POL60V and POL120V images respectively) and only then we estimated the Stokes parameters.

\section{Nuclear polarization measurements}

In Fig. 1 we show the images obtained with the $F 606 \mathrm{~W}+$ POLOV filter for our 9 targets. All these objects have been previously observed at different wavelengths with HST with several instruments and their morphology is already well studied. We only point out the presence of the optical nuclear sources, of which we aim to measure the polarization. Furthermore, a circumnuclear dusty disk is found in every galaxy, with the exception of 3C 274 where the gaseous disk is seen in emission; the optical jets of 3C 264 and 3C 274 already seen in previous HST images (Crane et al. 1993; Boksenberg et al. 1992) are also clearly detected.

In order to estimate the nuclear polarization of our targets we obtained photometry of each galaxy through synthetic apertures of increasing radii, up to 50 pixels, i.e. $\sim 11^{\prime \prime} 3$, centered at the galaxy's nucleus. In each aperture we measured the count rates in the 3 polarizers. These were scaled according to the prescription derived in the Appendix A and then combined to derive the Stokes parameters. In Fig. 2 we show the behaviour of the polarization parameters within each aperture for all 9 radio-galaxies.

The general behaviour is of a small percentage of polarization $P$ within the largest aperture, $P_{50} \lesssim 0.5 \%$ (and thus consistent with a null value given the calibration uncertainties) ${ }^{1}$ and a

1 with the exception of 3 C 274 for which $P_{50}=2.3 \%$, due to the presence of its bright and highly polarized jet. substantial increase of $P$ toward the center. The nuclear polarization measurements, $P_{3}$, are derived from an aperture of 3 pixels (as suggested by the same analysis performed on the polarization profiles on the standard stars) and are reported in Table 2. The percentage of polarization ranges from 1.2 to $7.5 \%$. These results represent a clear indication for the presence of a polarized component localized at the nucleus of each galaxy of the sample. Only for 3C 449 the nuclear polarization is marginally significant with $P_{3}=1.2 \pm 0.6 \%$. Apparently, there are no simple connections between the polarization properties (both percentage of polarization and position angle) of the FR I nuclei and other quantities, such as total and core radio-power, or orientation (derived from either core-dominance or dusty disk inclination).

These measurements refer to all light within the 3 pixels aperture, including the galaxy starlight. The presence of starlight (essentially unpolarized) causes a decrease with respect to the intrinsic level of polarization of the nuclear source.

Different approaches can be followed to remove the effects of this diluting component. The simplest method is to subtract from the total intensity a constant galaxy's background $I_{\text {gal }}$ measured in a circum-nuclear annulus (for which we chose a 0.1 width) immediately surrounding the nuclear point sources (see Fig. 3). The degree of polarization is then re-estimated as $P_{\text {nuc }}=$ $P_{3} \times I /\left(I-I_{\text {gal }}\right)$, while the position angle remains unchanged. The values of $P_{\text {nuc }}$ thus obtained are given in the third column of Table 2. They range from 1.9 to $11.3 \%$. In most cases the correction for dilution corresponds to an increase of the polarization by a relatively small factor. But for the nucleus with the lowest contrast against the galaxy's background, namely 3C 296, the correction for dilution is essential as it raises the nuclear polarization to $11.3 \%$ from an uncorrected value of $2.8 \%$.

This approach neglects the increase of surface brightness toward the center which, once modeled, would lead to a even higher nuclear polarization. However, all our targets are well reproduced by a power-law brightness profile with a small $(<0.3)$ logarithmic slope, and we found that this effect does not substantially affect our estimates.

A more subtle point is that so far we treated the starlight background as unpolarized. This is in general a good assumption, as shown by the low level of polarization at large radii shown by Fig. 2 which is consistent with a null value. Nonetheless, the presence of dusty disks (in all but one of our sources) suggests that dichroic polarization might be produced by the passage of light through these dust structures (we will return to this issue in Sect. 4). This might affect particularly the nuclei with the lowest polarization level and cause also a change in the polarization position angle. If the galaxy is polarized, the background must be subtracted from the nuclear aperture in the Stokes parameters space. We then estimated also the Stokes parameter $Q$ and $U$ within the region used to measure the galaxy's emission and we subtracted a polarized background. Only marginal differences are found with respect to the case of unpolarized background with changes in position angles always smaller than the statistical uncertainties.

We conclude that while the dilution from the galaxy starlight is quite significant in several galaxies, the final value for the nuclear polarization is rather robust against the various methods used to treat this effect.

We also note that an additional cause of dilution is the presence of (unpolarized) narrow-line emission. The presence of a Compact Emission Line Region (CELR), unresolved at 0.' 1 resolution, was shown by HST narrow-band imaging to be a characteristic feature of these low-luminosity radio-galaxies (Capetti et al. 2005). Measurement of the luminosity of the $\mathrm{H} \alpha+\mathrm{NII}$ lines 

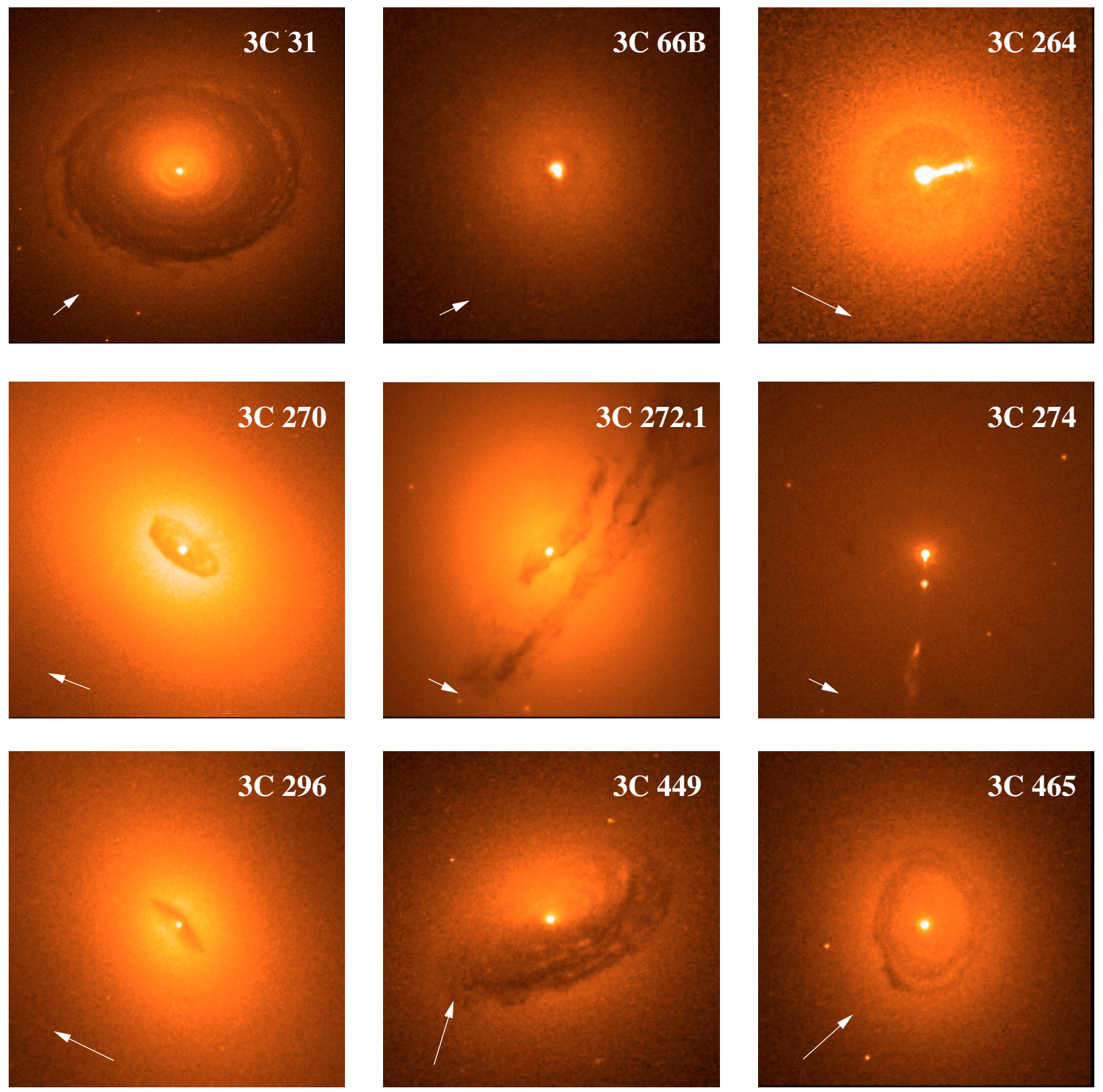

Fig. 1. ACS/HRC images of the 9 FR I radio-galaxies. The arrow in the bottom left corner is 1" long and points toward North.

(included in the spectral range covered by the $F 606 \mathrm{~W}$ filter) originating from the CELR is available for 8 galaxies discussed in this work, with the exception of 3C 296. The average equivalent width of these lines, measured against the nuclear sources, is $\sim 500 \AA$. This is substantially smaller than the $F 606 \mathrm{~W}$ filter width ( 2300 $\AA$ ) and this guarantees that the nuclear sources seen with the $F 606 W$ filter are dominated by continuum emission. We prefer not to correct the nuclear polarization on an individual basis, given the many sources of uncertainties related to this process, but this implies that the genuine nuclear polarization should be further increased to include also this diluting component.

\section{On the off-nuclear polarization}

\subsection{Polarization and dust structures}

In Fig. 4 we show the images of total intensity and of polarized flux for the 9 targets, obtained after a re-binning of $4 \times 4$ pixels, approximately to 0 . $^{\prime} 1 \times 0$.' 1 . In several cases regions of extended polarized emission (even leaving aside the polarized emission from the jets of 3C 264 a 3C 274) are detected, always associated with dust features, thus suggestive of dichroic polarization. The clearest cases are i) 3C 31 where polarization at a level of $2-4 \%$ is seen over a fan-shaped region corresponding the South-West side of its circumnuclear dusty disk; ii) $3 \mathrm{C} 465$ in which the Southern side of its dusty ring shows a polarization of 1-2\%; and iii) 3C 272.1, with $P \sim 1-1.5 \%$ over the filamentary dust structures East of the nucleus. In all cases the polarization is associated to the regions of higher absorption within the dusty structures. Marginal polarization, below a $1 \%$ level, might be present over the regions of higher absorption also in 3C 296 and 3C 449.

To put the connection between extinction and polarization on a firmer and quantitative ground in Fig. 5 are shown the images of total intensity, polarized flux, percentage of polarization, and extinction for 3C 31. The extinction map was obtained dividing 

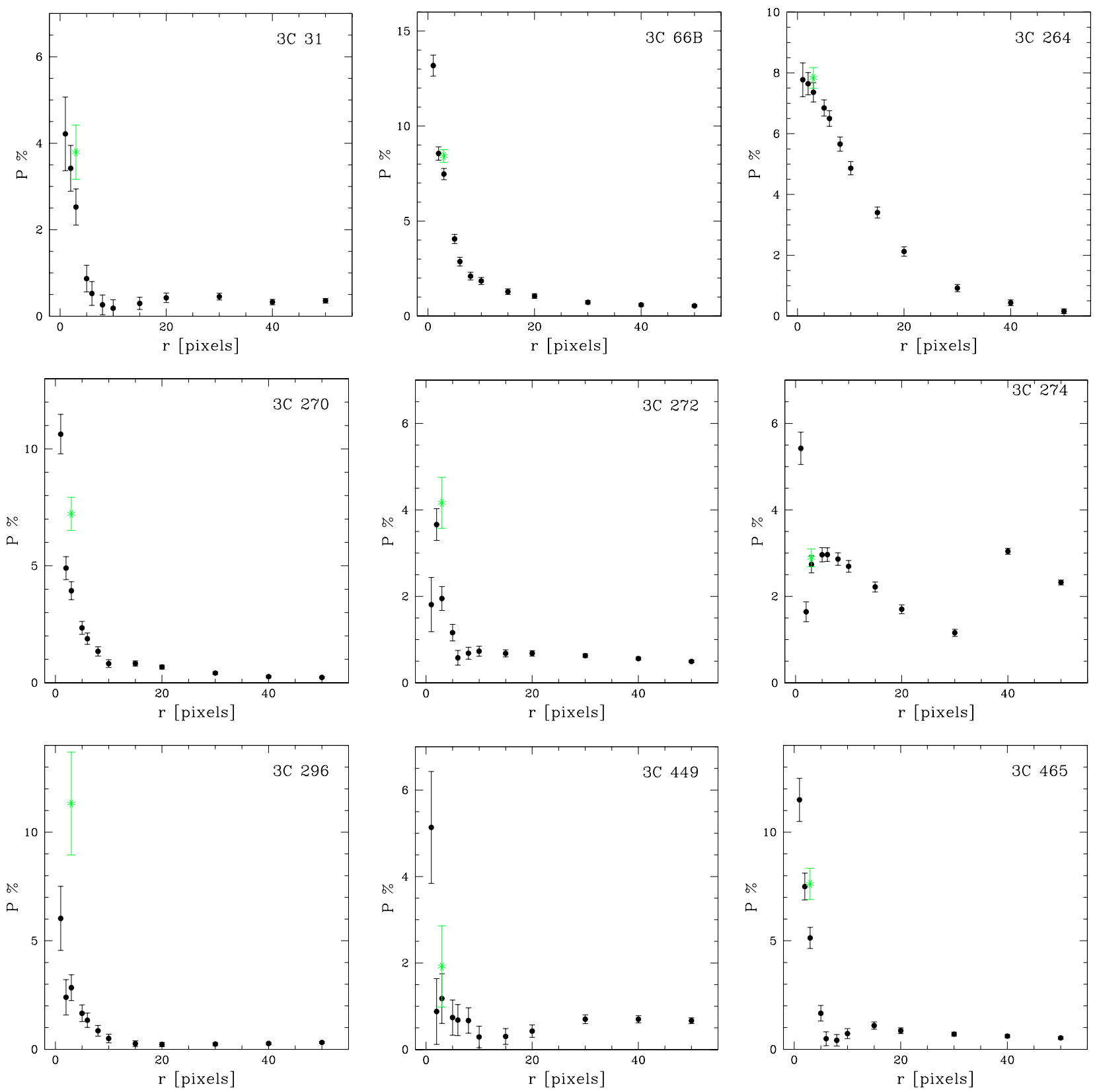

Fig. 2. Polarization measured through apertures of increasing radius $r$. The star at $r=3$ pixels marks the polarization level derived after correcting for the galaxy's dilution.

the HRC images by a NICMOS $H$-band $(F 160 W)$ image. This enables us to estimate the efficiency of the dichroic polarization. We find that, on average, a percentage of polarization of $\sim 2 \%$ corresponds to a color excess of $E(B-V) \sim 0.3$ having adopted the extinction law by Cardelli et al. (1989). The polarization efficiency is thus of $P / A_{\mathrm{V}} \sim 2 \% \mathrm{mag}^{-1}$ (referred now to the $V$ band). Slightly smaller estimates, $P / A_{\mathrm{V}} \sim 1.5 \% \mathrm{mag}^{-1}$, are obtained for 3C 272.1 and $3 \mathrm{C} 465$. These values should probably be considered as upper bounds since they were measured on the objects with the highest polarization. Furthermore, regions of relatively high extinction but with low polarization are also seen. Overall, these estimates compare favorably with the efficiency of dust interstellar polarization in the Galaxy, $P_{\max } / A_{\mathrm{V}} \sim 0.5-3 \% \mathrm{mag}^{-1}$ (Serkowski et al. 1975), and the $P / A_{\mathrm{V}} \sim 1 \% \mathrm{mag}^{-1}$ measured in the dust lane of Cen A (Hough et al. 1987; Schreier et al. 1996); however a more detailed analysis of the polarization efficiency and a comparison with other measurements requires multiband polarimetry as to determine its dependence with wavelength.

Considering the polarization vectors, in 3C 31 and 3C 465 their orientation is tangential with respect to the dusty disk (see Fig. 6, for the case of $3 \mathrm{C} 465$ ). This is what is expected when the magnetic field responsible for the grains alignment is stretched by differential rotation within the disk, leading to a toroidal magnetic configuration. In 3C 272.1 the polarization vectors are instead preferentially aligned with to the dust structures. This apparently different behavior might still be consistent with a tangential configuration of the magnetic field if the dust features are actually part of a dusty disk seen close to edge-on.

We cannot exclude the possibility that the observed regions of extended polarized emission are due instead to scattered nuclear light, an idea that is also supported by the orientation of the polarization vectors. However, this interpretation appears less 


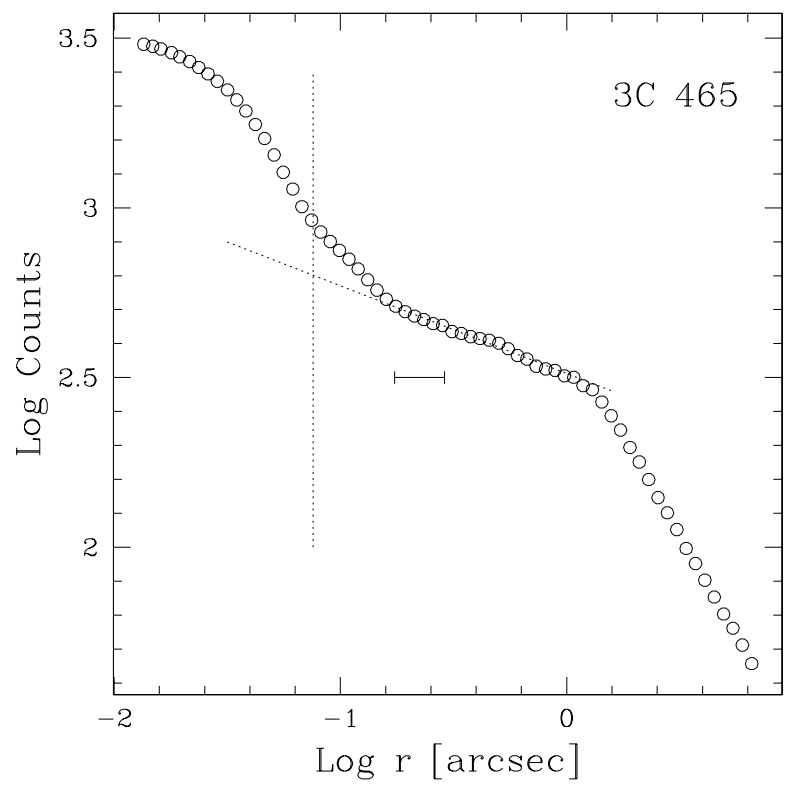

Fig. 3. Brightness profile of $3 \mathrm{C} 465$ used to estimate the dilution from the starlight emission. We marked the $0{ }^{\prime} 1$ annulus on which the galaxy's background has been estimated (horizontal bar), the radius of 3 pixels used for the nuclear aperture photometry (vertical dashed line) and the best fitting power-law to the nuclear slope of the brightness profile.

Table 2. Nuclear polarization.

\begin{tabular}{lccccc}
\hline \hline Name & $P_{3}$ & $P_{\text {nuc }}$ & PA & PA $_{r}$ & $\Delta \mathrm{PA}$ \\
\hline 3C 31 & $2.5 \pm 0.4$ & $3.8 \pm 0.6$ & $2.3 \pm 9.5$ & -18 & 20 \\
3C 66B & $7.5 \pm 0.3$ & $8.4 \pm 0.3$ & $79.6 \pm 2.3$ & 50 & 30 \\
3C 264 & $7.4 \pm 0.3$ & $7.8 \pm 0.3$ & $4.4 \pm 2.5$ & 30 & 26 \\
3C 270 & $3.9 \pm 0.4$ & $7.2 \pm 0.7$ & $172.5 \pm 5.6$ & -93 & 86 \\
3C 272.1 & $1.9 \pm 0.3$ & $4.2 \pm 0.6$ & $125.4 \pm 8.1$ & 1 & 56 \\
3C 274 & $2.7 \pm 0.2$ & $2.9 \pm 0.2$ & $103.0 \pm 4.1$ & -84 & 7 \\
3C 296 & $2.8 \pm 0.6$ & $11.3 \pm 2.4$ & $90.2 \pm 12.0$ & 40 & 50 \\
3C 449 & $1.2 \pm 0.6$ & $1.9 \pm 0.9$ & $115.3 \pm 27.9$ & 11 & 76 \\
3C 465 & $5.1 \pm 0.5$ & $7.6 \pm 0.7$ & $138.2 \pm 5.4$ & -49 & 7 \\
\hline
\end{tabular}

$P_{3}$ : polarization measured through a 3 pixels radius aperture; $P_{\text {nuc }}:$ nuclear polarization corrected for dilution of the galaxy's starlight. $\mathrm{PA}_{r}$ : radio jet position angle.

likely in view of the good spatial correspondence between extinction and polarization. In the case of scattering in a uniform circumnuclear disk, we would expect the polarized regions to trace the illumination pattern of the nuclear emission and, in case of an anisotropic radiation field, to be possibly enhanced along the direction of the radio-jets. This is not what is observed.

\subsection{Are there scattered nuclear radiation beams?}

Imaging polarimetry provides a tool to determine the geometry of the nuclear radiation field by measuring the large scale distribution of polarized light. We successfully used this technique for Seyfert galaxies (Capetti et al. 1995, 1996): around their nuclei we detected the characteristic centro-symmetric polarization pattern which arises from the scattering from a central source. The asymmetric distribution of polarized light enabled us to determine the angular extension of the region illuminated by the nucleus and to map the anisotropy of the nuclear radiation field.

This method is well suited in the case of FR I radio-galaxies. In fact, in the frame of the unification with BL Lac objects, they should harbour a collimated radiation beam which, unlike the case of BL Lac objects, is not pointing toward us. This beam might manifest itself through scattering, producing a collimated region of scattered light in the polarization images. Indeed HST imaging polarimetry of the FR I radio-galaxy Cen A (Capetti et al. 2000a) showed the presence of scattered nuclear radiation over an angular region which extends over $\sim 70^{\circ}$, centered on the radio-axis. If due to intrinsic anisotropy, and not to obscuring circumnuclear material, this implies the presence a less collimated nuclear radiation field than expected from a misdirected BL Lac beam with a large Lorentz factor.

We investigated whether a similar effect is seen in our 9 targets by exploring the azimuthal behaviour of the off-nuclear polarized light. The signature of scattered light from a mis-oriented beam should be an increase of polarization along the radio-axis, on both the jet and counter-jet side. This effect is not seen in any of the 9 radio-galaxies at a level of typically $0.5 \%$. As an illustrative example, in Fig. 7 we present the pattern of the polarization against the azimuthal angle for 3C 270.

Unfortunately, we cannot derive quantitative constraints from this upper limit, since this is the result of the interplay of several independent factors i.e. the intensity of the putative mis-directed beam, its ratio against the galaxy's starlight, and the optical depth of the scatterers. The detection of this effect in Cen A is probably not surprising due to the large amount of gas and dust in the circumnuclear regions of this object that provides a larger than average density of scatterers.

\section{Origin of nuclear polarization of low luminosity radio-galaxies}

The presence of polarized light can be associated with different physical processes: it can be intrinsic to the radiation mechanism, as in the case of synchrotron emission; alternatively, it can be due to the external factors, such as scattering or dichroic transmission through dust grains. In the following we examine which of these possibilities can explain the observed nuclear polarization in the LLRG, taking also into account previous results obtained from polarimetry of other classes of AGN.

\subsection{Dichroic polarization}

The presence of dust features surrounding the nuclei of most of our sources suggests that dichroic transmission might contribute to the nuclear polarization. In Sect. 4 we estimated an efficiency for the dichroic polarization of the off-nuclear dust features of $\sim 2 \% \mathrm{mag}^{-1}$ and this value should be considered as an upper bound since it was measured on the regions with the highest polarization. To account for the nuclear polarization with dichroic transmission, at least 1-6 mag of optical absorption are needed to produce the observed polarization. The presence of such a level of obscuration is expected to produce a significant reddening on the nuclei of our sources. We explored whether this is the case by comparing the nuclear spectral indices $\alpha_{\mathrm{UV}, \mathrm{O}}$ estimated in Chiaberge et al. (2002) using UV and optical observations, available for 7 of our sources ${ }^{2}$, with their degree of polarization. In case of dichroic polarization, we expect the steeper (redder) spectra to be associated with the more polarized nuclei. This is clearly not the case. The two nuclei with available UV data (in $3 \mathrm{C} 66 \mathrm{~B}$ and $3 \mathrm{C} 264$ ) with the highest polarization, $\sim 8 \%$,

\footnotetext{
${ }^{2}$ We also include an unpublished UV measurement for 3C 31 , obtained from an undergoing HST UV survey of the 3C sample (Prop. ID 10606, PI Sparks), corresponding to $\alpha_{\mathrm{UV}, \mathrm{O}}=1.9$.
} 

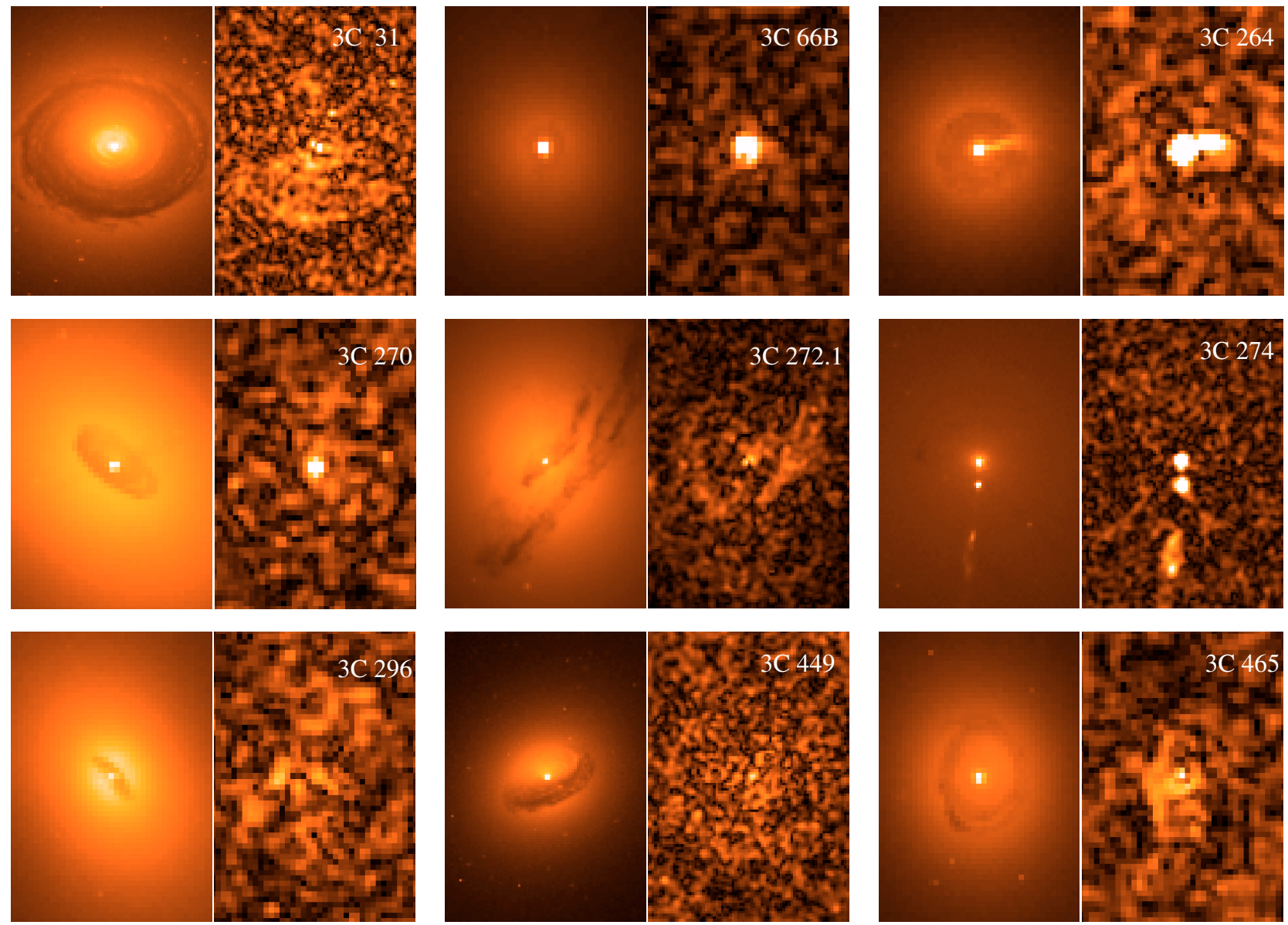

Fig. 4. Images of total intensity and polarized flux for the 9 radio-galaxies.

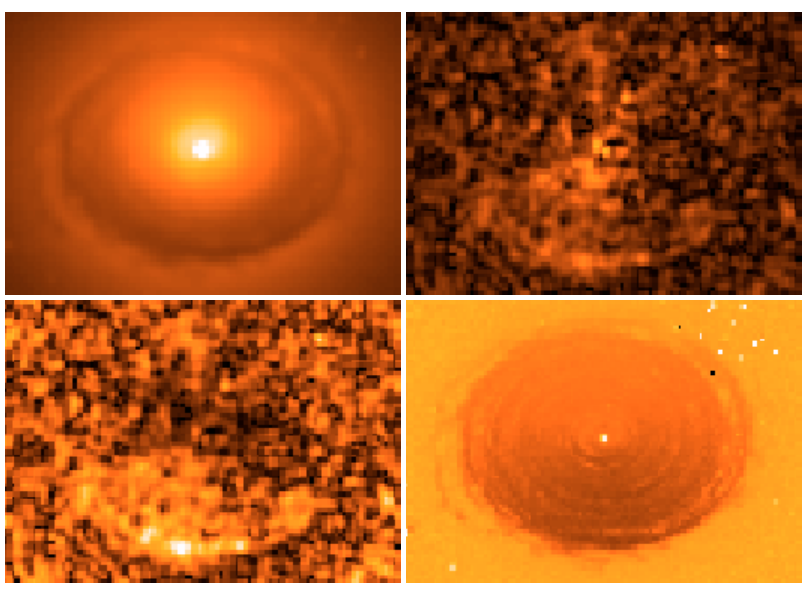

Fig. 5. Images of total intensity (top left), polarized flux (top right), percentage of polarization (bottom left), and extinction for 3C 31 (bottom right).

have the lowest values of $\alpha_{\mathrm{UV}, \mathrm{O}}$. Conversely, the steepest nucleus (in $3 \mathrm{C} 449$ ) has the lowest polarization. For a more quantitative analysis, we report in Fig. 8 the expected relationship between $P$ and $\alpha_{\mathrm{UV}, \mathrm{O}}$, for a dichroic polarization efficiency of $2 \% \mathrm{mag}^{-1}$. We also adopted a specific range of intrinsic spectral index, $\alpha_{\mathrm{UV}, \mathrm{O}}=1 \pm 0.5$ (as typical of extragalactic synchrotron sources, such as BL Lacs nuclei and extended jets, e.g. Fossati et al. 1998; Perlman et al. 2001) but the lack of connection

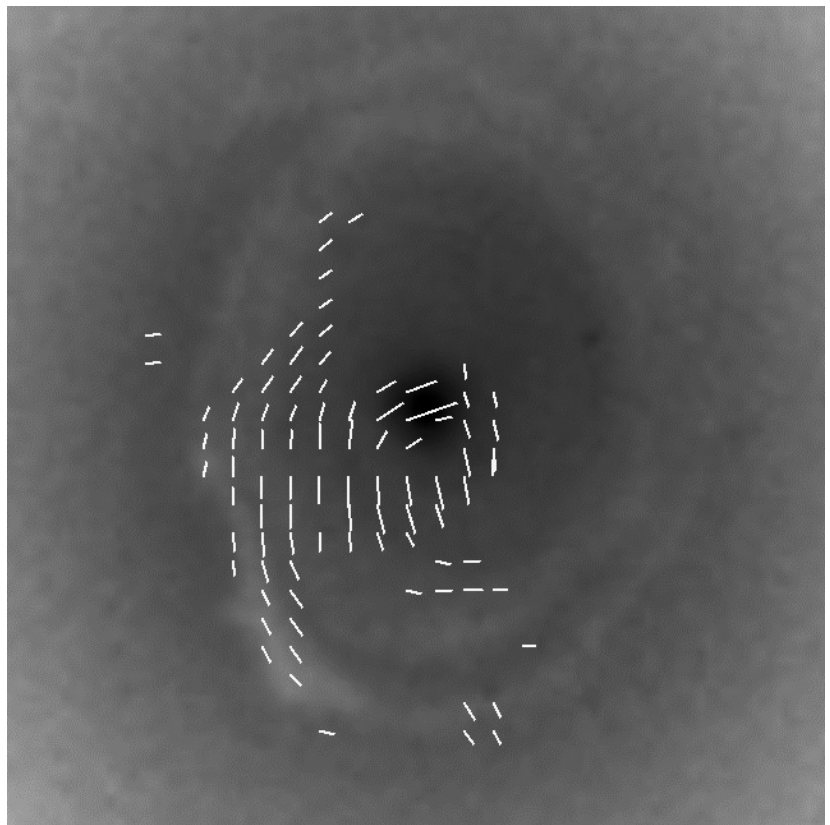

Fig. 6. Polarization vectors for $3 \mathrm{C} 465$ drawn at 0 ? $^{\prime} 1$ resolution in the regions where $P>1 \%$, superposed to the total intensity image.

between spectral index and polarization does not depend on this assumption. 


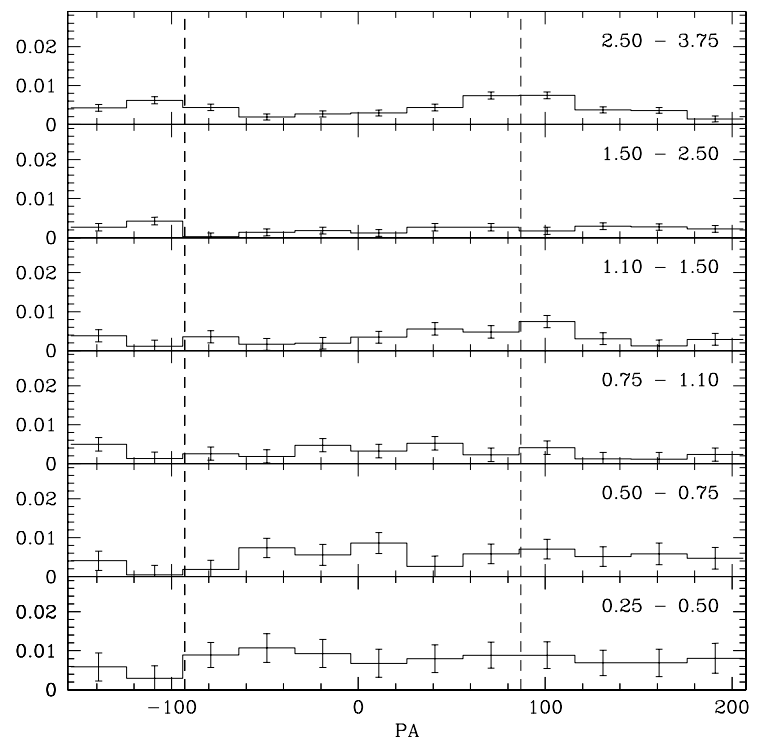

Fig. 7. Pattern of the polarization against the azimuthal angle (using the nucleus as origin) for $3 \mathrm{C} 270$. The polarization has been estimated over wedges of 30 degrees of width over 6 different ranges of radii, from 0.25 to $3^{\prime \prime} .75$, as reported in the top right corners of the panels. The dashed lines mark the position angles of jet and counter-jet. No polarization excess is found along the radio-jets axis.

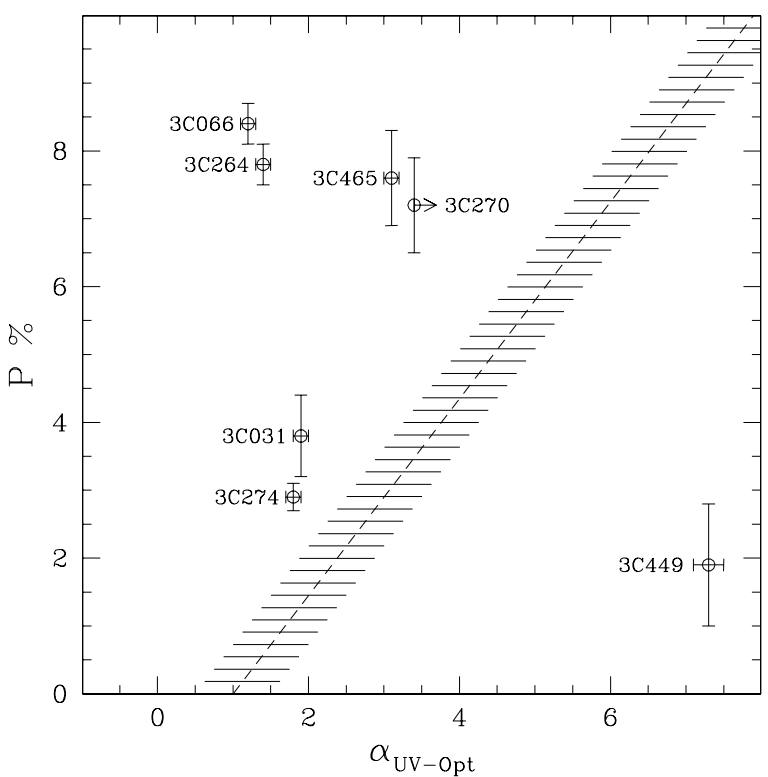

Fig. 8. UV to optical spectral indices against polarization of the FR I nuclei. The dashed line represents the relationship between these two quantities adopting a dichroic polarization efficiency of $2 \% \mathrm{mag}^{-1}$ and an intrinsic spectral index of 1 . The shaded region is the locus allowed by varying the un-absorbed spectral index by \pm 0.5 .

We conclude that dichroic polarization is unlikely to contribute significantly to the nuclear polarization, unless it corresponds to a very large range of dichroic efficiency, and extending to values far higher than measured in the dusty off-nuclear regions.

Note that the negligible level of polarization of the hosts also rules out the possibility of a significant contribution of interstellar Galactic polarization.

\subsection{Polarization from scattering}

\subsubsection{Case I: polar scattering from a hidden nucleus}

The clearer examples of polarized AGN associated to scattering are provided by type II Seyfert (Sy 2) galaxies and powerful narrow-lined radio-galaxies (e.g. Antonucci 1982, 1983; Cohen et al. 1999). Their polarized spectrum closely matches that of a type I (Sy 1) source, with the presence of a polarized featureless continuum and broad-lines, with levels of polarization that can be as high as $30 \%$. This is interpreted as due to scattering of light from an otherwise hidden nucleus. In these objects the polarization angle is generally well aligned with the perpendicular to the radio-axis (Antonucci 1983; Ulvestad \& Wilson 1984) most likely the same axis of the occulting torus, indicating that scattering takes place in a polar region located above the circumnuclear obscuring material.

The comparison between the position angle of the nuclear polarization and of the radio-jets thus provides a simple test to establish whether polar scattering might be at the origin of the observed polarization. The orientation of the radio-axes (and their offset from the nuclear polarization) for our sources are given in Table 2. They are taken from VLBI/VLBA observations (Xu et al. 2000; Venturi et al. 1995), except for 3C 296 for which we used the VLA maps by Hardcastle et al. (1997).

There is no preferential alignment between radio-axis and polarization, as the whole range of possible offsets (between 0 and 90 degrees) $\triangle P A$ is observed. In 5 galaxies (namely $3 \mathrm{C} 31$, $3 \mathrm{C} 66 \mathrm{~B}, 3 \mathrm{C} 264,3 \mathrm{C} 274$ and 3C 465) the polarization angles are parallel to the jet axis, to within 30 degrees, while for two sources (3C 270 and 3C 449) the nuclear polarization is close to perpendicular to the radio jets. For the remaining two galaxies (3C 272.1 and 3C 296) the offset is of $\sim 50$ degrees. These large misalignments from the perpendicularity expected in the case of polar scattering argue against this interpretation to account for the FR I nuclear polarization.

\subsubsection{Case II: equatorial scattering from a visible nucleus}

Type I Seyfert nuclei (and radio-quiet quasars) are also often polarized, although generally at significantly lower levels than Sy 2; their polarization angle is usually aligned with the radiojet, although there are examples of perpendicular polarization (Stockman et al. 1979; Antonucci 1983). This has been interpreted as a contribution to the polarized flux of scattered light from an equatorial disk-like region, which is hidden in the case of Sy 2 (Smith et al. 2004). The relative weight of the polar vs. equatorial scattering sets the dominance of polarization perpendicular or parallel to the AGN symmetry axis. Thus, in the case of scattering from a visible nucleus, there are no simple predictions for the orientation of the polarization. The analysis of a sample of 36 type I Seyfert (Smith et al. 2002) gives a median polarization of $0.5 \%$, with only one object above a level of $2 \%$. The significantly lower polarization level in Sy 1, with respect to type 2 , is naturally accounted since in this situation the light from the unocculted nucleus provides a copious source of diluting unpolarized light.

More quantitatively the level of nuclear polarization is set by: i) the optical depth $\tau$ of the scattering region; and ii) the covering angle of the scattering material $\Omega$ (that together determine the fraction of scattered nuclear light); but the observed degree of polarization depends also on the geometry of the system i.e.; iii) on the scattering angle; and iv) on the geometrical cancellation produced by summing over polarization vectors with 


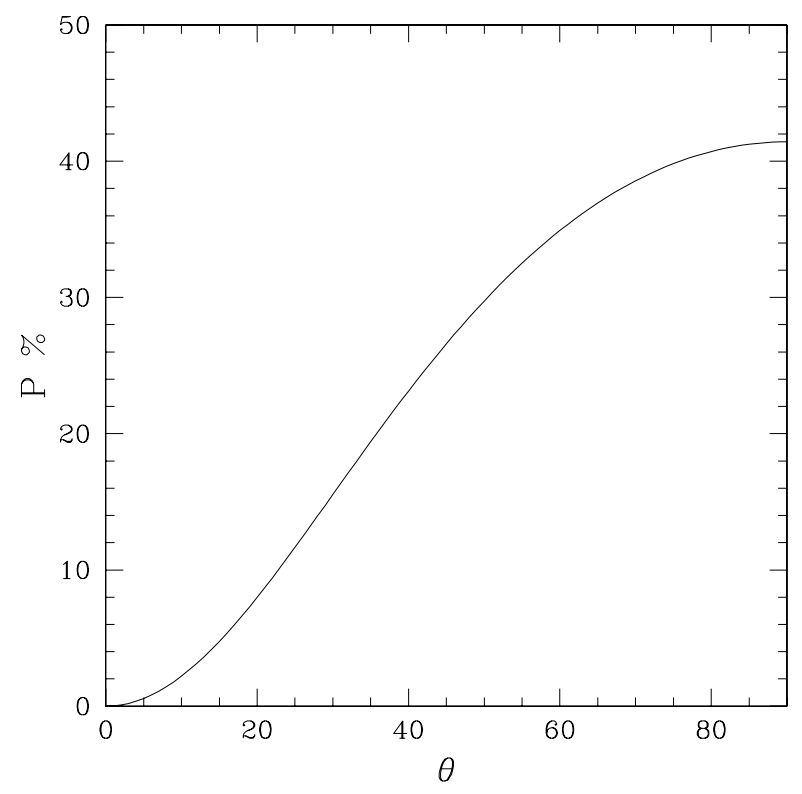

Fig. 9. Dependence of the degree of polarization of light scattered in an optically (and geometrically) thin circumnuclear disk on the orientation of the disk axis with respect to the line of sight. 90 degrees corresponds to an edge-on disk.

different orientation within the scattering region. In Fig. 9 we show the dependence on orientation of the fractional polarization of the scattered light in a thin disk. The optimal geometrical setting to produce a high polarization is an edge-on thin disk (Smith et al. 2002) that corresponds to a degree of polarization of $f \sim 40 \%$ (while the average for a randomly oriented sample is $f \sim 30 \%)$. The nuclear polarization level is then given by $P=f \Omega\left(1-\mathrm{e}^{-\tau}\right)$. Our observations provided measurements in the range $\sim 2-11 \%$ with a median value of $7 \%$, more than an order of magnitude higher than observed in Seyfert 1. In order to achieve this level of polarization an implausible high fraction of nuclear light $\left(\Omega\left(1-\mathrm{e}^{-\tau}\right) \gtrsim 0.2\right)$ is needed to be scattered into our line of sight.

\subsection{Synchrotron emission}

Synchrotron emission is intrinsically polarized and it has been firmly established that it represents the dominant emission process associated to several classes of AGN. In particular this is the case of all objects falling within the definition of Blazars, including BL Lac objects and core-dominated radio-loud quasars, that are indeed highly polarized (e.g. Angel \& Stockman 1980).

In the framework of the most promising unifying model for weak radio-loud AGN, LLRG are identified as the parent (misdirected) population of BL Lac objects (e.g. Urry \& Padovani 1995, for a review). The non-thermal continuum emission of BL Lacs results from the observation of plasma moving at relativistic speed at a small angle with respect to its direction of motion (Blandford \& Rees 1978). This emission is expected to be present also in radio-galaxies although not amplified by relativistic beaming, or even de-amplified.

Here we then compare the polarization properties of LLRG and BL Lacs. Such a comparison is complicated by the high variability of BL Lacs polarization and its dependence on wavelength (Brindle et al. 1986), by the effects of different viewing angle and relativistic aberration. Nonetheless, we find that the degree of polarization of BL Lac objects is quantitatively similar to the measurements presented here for LLRG: for example, the median value of polarization in the sample of 20 radio-selected BL Lacs studied by Impey \& Tapia (1990) is $\sim 8 \%$, while X-ray selected BL Lacs are apparently slightly less polarized (Jannuzi et al. 1994). Concerning the polarization angle, Gabuzda et al. (2006) showed that BL Lac have a close alignment between radio and optical polarization; conversely, although the radio polarization is preferentially aligned with the jet direction (within 20 degrees), a smaller but substantial population shows polarization orthogonal to the jet axis (within 30 degrees). Again, this is analogous to the situation found for LLRG.

We conclude that the polarization properties of LLRG nuclei closely resemble those of BL Lac objects, strongly favoring the interpretation that they are dominated by synchrotron emission.

The direct comparison between the optical and radio core polarization properties of our radio-galaxies is in general hampered by the lack of high quality radio data. Nonetheless, Kharb et al. (2005) provide VLBI measurements of the radio core polarization of 3 galaxies of our sample (namely 3C 66B, 3C 264 and $3 \mathrm{C} 270$ ). In all 3 cases the percentage of polarization is quite low (between 0.4 and $1.1 \%$ ) and much lower than measured in the optical where we measured typically a $8 \%$ level. This is in line with the result we obtained for Cen A (Capetti et al. 2000a), where a $11 \%$ near infrared nuclear polarization is contrasted by a $0.46 \%$ of radio core polarization (Burns et al. 1983).

The most likely explanation for the different polarization level of the radio and optical nuclei is the presence of a depolarizing medium located in front of the active nucleus. Evidence for circumnuclear gas in FR I nuclei came recently from X-ray observations of these objects (Balmaverde et al. 2006); we detected nuclear X-ray absorption in 4 galaxies at a level of $N_{\mathrm{H}} \sim$ $(0.2-5) \times 10^{22} \mathrm{~cm}^{-2}$. X-ray absorbed sources are associated with the presence of highly inclined dusty disks seen in the HST images. This suggests the existence of a flattened X-ray absorber, but of much lower optical depth than in classical obscuring tori. Due to the $\lambda^{2}$ dependence of Faraday rotation, this gas might act as a depolarizing screen onto the nucleus in the radio band, while leaving unaltered the optical polarization level.

\subsection{Polarized emission from radiatively inefficient accretion flows}

In the introduction we discussed the possibility that the faint optical nuclei detected in FR I radio-galaxies might originate from a low radiative efficency accretion flow. Is this consistent with our polarization measures? Indeed, recent observations indicate that also the emission associated with the black hole at the center of our own Galaxy - a likely candidate system for such accretion regime - produces polarized light outside the radio-band. In fact Sgr A* showed high polarization (up to $40 \%$ ) associated to flares in the $\mathrm{K}$ band (Trippe et al. 2006). This might be taken as an indication that accretion in such conditions is able to produce significant polarization, opening an alternative explanation to the association with the jet emission we proposed.

The low-frequency emission expected from such flows originates as cyclo-synchrotron emission from thermal plasma, which is expected to be polarized. Given the temperature, density and magnetic energy density conditions theoretically inferred, this spectral component should dominate - at least in the simplest scenario - at, typically, mm wavelengths (e.g. Mahadevan 1997).

Nonetheless, depending on the physical parameters describing a RIAF, the polarized cyclo-synchrotron radiation might still dominate, over Comptonization and bremsstrahlung emission, also at higher frequencies. While no unique prediction is 
possible, in this situation, the emission in the optical band would be typically characterized by a steep spectrum. This possibility is then disfavored by the flat spectral energy distribution of FR I nuclei in the UV-optical band found by Chiaberge et al. (2002).

The situation could be more complicate. The very presence of rapidly varying polarized IR (and X-ray) emission in Sgr A* have led to the hypothesis that flaring is associated to nonthermal synchrotron radiation, from a dissipation region either in the jet or within the accreting flow. Apparently, this also leaves the possibility of RIAF emission open also for FR I. However, it should be stressed that while high levels of polarization in $\mathrm{Sgr} \mathrm{A}^{*}$ are observed during flares, the detection of nuclear polarization in 8 out 9 of our FR I sources (leaving aside the $2 \sigma$ detection in 3C 449) argues for a more stable process.

\section{Summary and discussion}

We obtained optical imaging polarimetry with the ACS/HRC aboard the HST of the 9 closest radio-galaxies in the $3 \mathrm{C}$ catalogue with an FR I morphology. The analysis of the observations of standard polarization targets lead to a calibration of the ACS/HRC polarimeter accurate to a level of $0.4 \%$.

The nuclear sources seen in direct HST images in these galaxies are found to be polarized with levels in the range $\sim 2-11 \%$ with a median value of $7 \%$. There is no clear preferential alignment between radio-axis and polarization vectors; the whole range of possible offsets is observed, with only a mild preference (in 5 objects) for polarization parallel to within $30 \mathrm{de}$ grees to the radio-jets, but cases of perpendicular polarization are also found, similar to the case of the nuclear polarization of Cen A.

In order to explore the origin of the nuclear polarization, we discussed the different mechanisms that produce polarized emission and conclude that the most likely interpretation is a synchrotron origin for the optical nuclei. In fact, polarization induced by dichroic transmission predicts that the most polarized nuclei should be the most absorbed, while their UV-optical spectral slope indicates that this is not the case. The possibility of polarization produced by polar scattering of light from an obscured nucleus (similarly to what is observed in type II Seyfert and narrow-line radio-galaxies) contrasts with the lack of perpendicularity between radio-jets and nuclear polarization. Scattering occurs also in type I AGN, but in this case the unocculted nucleus provides a copious source of unpolarized light, and indeed their typical polarization is of only $\sim 0.5 \%$. In order to achieve the level of polarization measured in LLRG an implausible high fraction of nuclear light $(\gtrsim 0.2)$ should be scattered into our line of sight.

This leaves us with the sole possibility that the nuclear light in FR I is intrinsically polarized, such as in the case of synchrotron emission. This idea is strengthened by the analogy with the polarization properties of BL Lac objects (from the point of view of percentage of polarization and of the complex relationship between polarization position angle and radiojet axis) where the dominant contribution of synchrotron light is well established. Incidentally, the similarity of LLRG nuclei and BL Lacs polarization adds to the list of properties shared by these two classes of objects, strengthening the case in favour of the FR I/BL Lac unified model.

Polarized emission is also expected in case of low radiative efficency accretion flows, although restricted to the spectral region dominated by cyclo-synchrotron emission. It is possible to ascribe the optical polarization to this process only if the cyclosynchrotron component, peaking in the $\mathrm{mm}$ region, remains the dominant radiation process into the optical band. While this cannot be excluded on purely theoretical ground, in such case the optical light would represent an exponential emission tail and it should show a very steep spectrum, contrarily to what is seen in FR I nuclei. Similarly, the presence of rapidly varying polarized IR emission in Sgr A have lead to the hypothesis that (at least) flaring is associated to non-thermal synchrotron radiation. However, it should be stressed that polarization in Sgr A* is only observed during flares, while the detection of nuclear polarization in the FR I sources argues for a more stable process.

These results, taken together, lead us to the conclusion that the optical emission of FR I nuclei is most likely dominated by synchrotron emission from the base of their jets.

This conclusion relies on the absence of an additional nuclear component, hidden to our view, other and (if it has to alter considerably our findings) substantially brighter than the synchrotron nuclei. The results presented here tell us that we are not seeing compact scattering regions illuminated by a bright nucleus, but the existence of a bright, hidden, nuclear source can not be ruled out on the base of the present data alone.

However, this possibility sets the following requirements on the nuclear structure of these radio-galaxies: i) the putative extra component must be hidden to our view in the optical band; since our sample is unbiased with respect to orientation, the occulting material should essentially take the form of a sphere, with, necessarily, only a free path coincident with the radio-jets; ii) it must be hidden also in the X-ray band; Evans et al. (2006) explored this possibility and concluded that this is incompatible with X-ray observations of FR I sources unless they are covered by extreme gas column densities, with $N_{\mathrm{H}}>10^{24} \mathrm{~cm}^{-2}$, far higher than in FR II radio-galaxies; iii) the optical depth toward the true nucleus must be also extremely high in the midinfrared to prevent the view of the nuclear emission reprocessed by the circumnuclear dust; in fact 3C 274 does not show a substantial MIR excess with respect to the extrapolation of the synchrotron component to this band (Whysong \& Antonucci 2004) and Spitzer observations of another 4 objects of our sample indicates an overall low activity level (Ogle et al. 2006), in line with previous findings based on ISO data (Müller et al. 2004); iv) the correlation between the optical nuclear continuum and the luminosity of the emission lines in these radio-galaxies suggests that the Narrow Line Region gas is ionized by the jet emission (Capetti et al. 2005); apparently, also the NLR gas does not see any additional ionizing photon field; v) since the jet component is visible in both optical and X-ray bands, it must originate outside the obscuring structure. We conclude that, most likely, the non-thermal emission represents the dominant radiative manifestation in the FR I nuclei.

The association between optical nuclei and jet emission was already suggested by Chiaberge et al. (1999) and Balmaverde et al. (2006) based on the close correlation between radio and optical nuclear luminosities and it is fully supported by the present polarimetric analysis. The radio/optical/X-ray correlations recently found by Balmaverde \& Capetti (2006) in a sample of radio-loud early-type galaxies extend this connection toward lower power, down to radio luminosities $v L_{r} \sim 10^{36} \mathrm{erg} \mathrm{s}^{-1}$ and to optical luminosities as low as $\sim 10^{38} \mathrm{erg} \mathrm{s}^{-1}$. The reasonable (and testable) extension of these polarimetric results to this latter sample would set a limit to any emission from the accretion process as low as $L / L_{\text {Edd }} \lesssim 10^{-9}$ in the optical and X-ray bands. In contrast, as already discussed in Capetti \& Balmaverde (2006), their Bondi accretion rates, $\dot{M}_{\mathrm{B}}$, derived from Chandra 
observations (e.g. Pellegrini 2005) are relatively large, $\dot{M}_{\mathrm{B}} / \dot{M}_{\mathrm{Edd}} \sim 10^{-2}-10^{-4}$. This raises the possibility that most of the accreting gas fails to reach the central object, possibly carried away by outflows originating at relatively large radii. These findings represent a challenge to the models of jet production, as they require that the energy released by gas accretion onto the supermassive black-hole is channeled with high efficiency in jet mechanical energy while leaving the accretion disk in a extremely low radiative state.

In addition to the polarization in the nuclei and from the large scale optical jets (which are presented elsewhere, Perlman et al. 2006), regions of extended polarized emission are also detected. They are closely cospatial with dust features (mainly taking the form of dusty circumnuclear disks) indicative of polarization due to dichroic transmission. In the two clearest examples the polarization vectors are tangential to the disks as expected when the magnetic field responsible for the grains alignment is stretched by differential rotation.

Conversely, we fail to detect an extended component of polarized light aligned with the radio-jet axis. This component might have been expected in the frame of the unification of FR I with BL Lac objects, since FR I should harbour a collimated radiation beam misaligned with respect to our line of sight that might be visible in scattered light. Indeed, this effect is present in the radio-galaxy Cen A that shows the characteristic centrosymmetric polarization pattern centered on the radio-axis. We did not find the polarimetric signature of this effect in any of the galaxies of the present sample, with limits to the excess of polarized light along the radio-axis at a level $\lessgtr 0.5 \%$. We cannot derive quantitative constraints from this upper limit as it depends on the intensity of the putative mis-directed beam (with respect to the galaxy's starlight) but also on the optical depth of the scatterers. Thus, our the results are inconclusive on whether a radiation misaligned beam is present in FR I.

\section{Appendix A: The ACS/HRC polarimeter}

Several issues concerning the use of the HRC as a polarimeter must be addressed before deriving the polarization properties of scientific targets, in particular related to the possible presence of instrumental polarization. They have been discussed in detail in Biretta et al. (2004) and are here only briefly summarized. Instrumental polarization might arise due to the presence of mirrors with large incident angles and of strongly tilted CCD detectors. The first point is particularly important since light reflected by mirrors may be affected by both di-attenuation (instrumental polarization caused by the different reflectivity for light polarized parallel or perpendicular to the incidence angle) and phaseretardance (the conversion of linear into elliptical polarization). These result in an instrumental polarization that varies with the telescope orientation as well as with the polarization state of the incident light. In addition, the polarization measurements are affected by i) the accuracy of the flat-field in the individual polarizers; ii) the polarizers orientation, which might differ from the nominal value; and iii) by the polarizers relative transmission.

Here we present the results obtained concerning the presence of instrumental polarization based on the analysis of the calibration observations of standard polarimetric stars limiting to our filter/instrument combination i.e. $\mathrm{HRC}+F 606 \mathrm{~W}$.

Two unpolarized standard stars, GD319 and G191B2B, lead to measurements of the count rates through each of the 3 polarizers (using an aperture of 50 pixels), see Table A.1. They indicate a substantial departure from an ideal polarimeter, for which one would expect equal count rates. Conversely, these observations lead to a measurement of the relative ratios of POL60V/POL0V $=1.095 \pm 0.004$ and POL120V/POL0V $=$ $1.109 \pm 0.004$, where we used the average values from the 3 observations (GD319 was observed twice). The individual ratios measurements differ by less than $0.3 \%$, a level similar to the statistical uncertainties.

We then estimated the count rates for the available observations of a polarized standard star, BD+64D106. It was observed at 5 different epochs and, most importantly for our discussion, at 5 different telescope roll angles. We again derived the count rates which are presented in Table A.1. Different exposures were not combined, but treated individually, for a total of 8 datasets.

We followed two methods to derive the polarization parameters of $\mathrm{BD}+64 \mathrm{D} 106$ from these observations. In the first approach we ascribed the different count rates measured for the unpolarized standards to a difference in the transmission of the 3 polarizers. We then simply scaled the count rates for BD+64D106, by 1.095 and 1.109 in the POL60V and POL120V images respectively, and only then we estimated the Stokes parameters. In Table A.1 we present the resulting values of $P$ and PA. The averaged values are $P=5.1 \pm 0.4 \%$ and $\mathrm{PA}=97.3 \pm 1.5$ (the errors quoted are the rms of the individual measurements) in very close agreement with the ground based measurements of this star in the $R$ band, $P=5.150 \pm 0.098$ at PA $96.74 \pm 0.54$ (Schmidt et al. 1992).

In the second method we corrected all measurements ascribing the count rates differences to the presence of instrumental polarization. The count rates measured for the unpolarized stars correspond to an instrumental polarization with Stokes parameter $Q=-6.36 \%$ and $U=0.76 \%$ (in the instrument reference frame). We estimated the "raw" Stokes parameters, then removed the instrumental polarization in the Stokes parameter space from all measurements. The resulting polarization parameters, $P=4.8 \pm 1.2 \%$ and $\mathrm{PA}=100.1 \pm 4.9$, are still in a reasonable agreement with the known polarization of BD+64D106, but they do not match as closely as those derived from the first method. Furthermore, they show a larger dispersion as well as substantial changes related to the telescope roll angle, unlike what we found from the first method.

But a better insight into these issues comes from combining opportunely the observations obtained at PAV3 $=-76,-16$ and 44 . These observations were planned to be separated by exactly 60 degrees in the telescope roll angle. Combining the images taken through one given polarizer at the three different roll angles, it is equivalent to the situation of observing the target through three polarizers separated by 60 degrees at the same roll angle. It is also worth noting that by rolling the telescope one rotates any instrumental polarization in the Equatorial system enabling us to determine it from observations of standard stars at different rolls. It is thus possible to measure the polarization parameters, removing the uncertainty due to the filter transmission. In particular, the ratio between the total intensities is a direct measure of the relative filter transmission. We obtained $I 60 / I 0=1.092 \pm 0.002$ and $I 120 / I 0=1.102 \pm 0.002$, having indicated with $I 0, I 60$ and $I 120$ the total intensity derived combining the observations at three roll angles of POL0V, POL60V and POL120V respectively. These values differ only marginally, and within the statistical uncertainties, from the ratios estimated from the unpolarized stars, i.e. 1.095 and 1.109.

This strongly supports the interpretation that the dominant contribution to the observed polarization level for the unpolarized standard stars comes from differences in the filter transmission. By applying a simple correction to the count rates we are able to reproduce accurately the expected values for the 
Table A.1. Standard stars observations.

\begin{tabular}{lccccccccc}
\hline \hline Star & Comment & \multicolumn{3}{c}{ Count rates } & \multicolumn{3}{c}{ Method I } & \multicolumn{3}{c}{ Method II } \\
& & PAV3 & POL0V & POL60V & POL120V & $P \%$ & PA & $P \%$ & PA \\
\hline GD319 & unpol. & 318 & 58906 & 64230 & 65150 & $0.2 \pm 0.6$ & - & $0.2 \pm 0.6$ & - \\
GD319 & unpol. & 276 & 58806 & 64561 & 65300 & $0.2 \pm 0.6$ & - & $0.6 \pm 0.6$ & - \\
G191B2B & unpol. & 91 & 138556 & 151884 & 153381 & $0.1 \pm 0.4$ & - & $0.5 \pm 0.4$ & - \\
BD+64D106 & pol. & 89 & 607846 & 723703 & 709541 & $4.9 \pm 0.2$ & $97.3 \pm 2.1$ & $4.3 \pm 0.2$ & $106.0 \pm 2.4$ \\
BD+64D106 & pol. & 135 & 651915 & 716861 & 662958 & $5.8 \pm 0.2$ & $97.1 \pm 1.7$ & $4.6 \pm 0.2$ & $93.7 \pm 2.2$ \\
BD+64D106 & pol. & -76 & 647274 & 760131 & 721303 & $4.6 \pm 0.2$ & $96.2 \pm 2.2$ & $3.4 \pm 0.2$ & $102.1 \pm 3.0$ \\
BD+64D106 & pol. & -76 & 645337 & 760477 & 719661 & $4.8 \pm 0.2$ & $96.3 \pm 2.1$ & $3.6 \pm 0.2$ & $101.9 \pm 2.8$ \\
BD+64D106 & pol. & -76 & 649304 & 769996 & 730473 & $5.0 \pm 0.2$ & $99.1 \pm 2.0$ & $3.9 \pm 0.2$ & $105.1 \pm 2.6$ \\
BD+64D106 & pol. & -16 & 704965 & 718297 & 713385 & $5.6 \pm 0.2$ & $100.1 \pm 1.7$ & $5.4 \pm 0.2$ & $92.5 \pm 1.8$ \\
BD+64D106 & pol. & 44 & 666275 & 726582 & 790493 & $4.7 \pm 0.2$ & $95.8 \pm 2.1$ & $6.2 \pm 0.2$ & $99.5 \pm 1.6$ \\
BD+64D106 & pol. & 44 & 663241 & 720569 & 791937 & $5.3 \pm 0.2$ & $96.8 \pm 1.9$ & $6.8 \pm 0.2$ & $100.0 \pm 1.5$ \\
\hline BD+64D106 & \multicolumn{9}{c}{ Averaged values / rms } \\
\hline
\end{tabular}

polarized standard star. The resulting dispersion in the polarization degree is $0.4 \%$, with PA variations of less than 2 degrees. These values can be used as estimates for the uncertainties due to any residual instrumental polarization as well as to the flat field accuracy on the polarization measurements for our science targets (in addition to the statistical uncertainties).

The observations of the unpolarized standard stars can also be used to test if there are any differences between the point spread function produced by each polarizers. This is important since we calibrated our polarization measurements using large apertures (50 pixels). However, since for our science targets we are interested in measuring the nuclear polarization we will be forced to use substantially smaller apertures. If, on a scale of a few pixels, there are differences in the PSF of each polarizer, we might obtain spurious polarization measurements.

To test this effect, we measured the polarization parameters using the count rates integrated through several synthetic apertures, ranging from a radius of 1 pixel to 50 pixels. The derived levels of polarization are shown in Fig. A.1. The polarization is below a $0.4 \%$ level down to a radius of 3 pixels. This is consistent with a null value within the errors due to the polarization calibration and with the statistical uncertainties. Conversely, using even smaller apertures, the polarization level increases to $\sim 1 \%$ at 2 pixels and even larger values using a 1 pixel aperture. Since we are dealing with unpolarized stars, this is a spurious polarization signal, most likely to be ascribed to a combination of different PSF and possibly also to small mis-centering of the stars. This indicates that robust polarimetric measurements can be obtained with synthetic apertures of radius as small as $r \gtrsim$ 3 pixels, with no appreciable differences in accuracy with respect to those used for the polarimetric calibrations that were derived from large apertures.

We finally note that the lack of sufficient calibration data in the UV band prevents us from following the strategy adopted for the optical data and from analyzing the data for the two radiogalaxies observed in this band.

\section{Appendix B: On the bias in the polarization measurements}

It is well known that linear polarization measurements are affected by a bias due to the fact the $P$ is a definitive positive quantity. Consequently, it follows a Rice (and not a Gaussian) probability distribution (e.g. Wardle \& Kronberg 1974).

The resulting effect is that the degree of polarization is overestimated at low signal-to-noise ratios. E.g., a null level of polarization corresponds to an average measurement of $P=\sigma_{P}$.
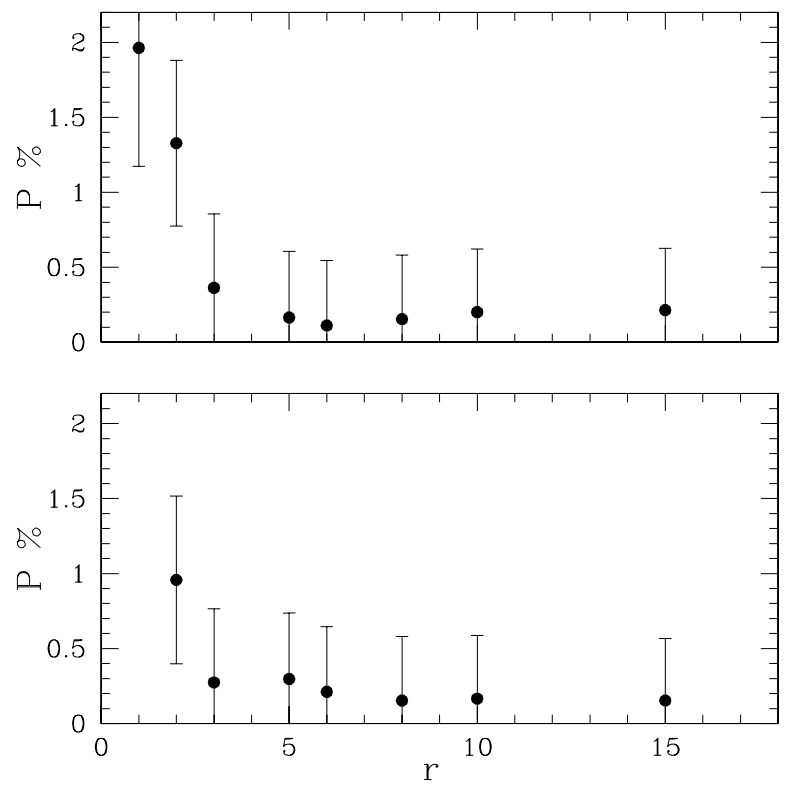

Fig. A.1. Percentage of polarization measured from two sets of observations of an unpolarized standard star. Different circular synthetic apertures were used. The polarization level is consistent with a null level down to an aperture of 3 pixels. Only below this radius small differences between the Point Spread Function through the polarizers produce a spurious polarization.

This is clearly seen in Fig. A.1 where the level of polarization for the unpolarized stars decreases at large radii to $\sim 0.2 \%$, approximately at a $1 \sigma$ level. A similar behaviour is found in the azimuthal behaviour of the off-nuclear polarized light (Fig. 7) where the minimum polarization is $\sim 0.5 \%$. This effect becomes rapidly less important at higher signal-to-noise ratios. In fact, the polarization overestimate is already reduced to a factor of $\sim 1.1$ at $P=2 \times \sigma_{P}$ and becomes essentially negligible at higher significance level.

Several methods to de-bias polarization data have been proposed (e.g. Simmons \& Stewart 1985) based on the probability distribution of the errors in the Stokes parameters. However, such an approach requires that the measurement errors are dominated by statistics. This is not necessarily the case for our measurements in which a possible contribution of instrumental polarization might be present. We therefore preferred not to attempt any correction for the polarization bias and decided to consider in our discussion only regions of sufficiently high polarization, $P>2 \times \sigma_{P}$, not significantly affected by this bias. 


\section{References}

Angel, J. R. P., \& Stockman, H. S. 1980, ARA\&A, 18, 321

Antonucci, R. R. J. 1982, Nature, 299, 605

Antonucci, R. R. J. 1983, Nature, 303, 158

Balmaverde, B., \& Capetti, A. 2006, A\&A, 447, 97 (BC06)

Balmaverde, B., Capetti, A., \& Grandi, P. 2006, A\&A, 451, 35

Biretta, J. A., \& Kozhurina-Platais, V. 2004, Instrument Science Report ACS 2004-10 (Baltimore: Space Telescope Science Institute)

Biretta, J. A., Kozhurina-Platais, V., Boffi, F., Sparks, W. B., \& Walsh, J. 2004, Instrument Science Report ACS 2004-09 (Baltimore: Space Telescope Science Institute)

Boksenberg, A., Macchetto, F., Albrecht, R., et al. 1992, A\&A, 261, 393

Brindle, C., Hough, J. H., Bailey, J. A., Axon, D. J., \& Hyland, A. R. 1986, MNRAS, 221, 739

Burns, J. O., Feigelson, E. D., \& Schreier, E. J. 1983, ApJ, 273, 128

Capetti, A., Axon, D. J., Macchetto, F., Sparks, W. B., \& Boksenberg, A. 1996, ApJ, 466, 169

Capetti, A., \& Balmaverde, B. 2006, A\&A, 453, 27 (CB06)

Capetti, A., Macchetto, F., Axon, D. J., Sparks, W. B., \& Boksenberg, A. 1995, ApJ, 452, L87

Capetti, A., Macchetto, F. D., Sparks, W. B., \& Biretta, J. A. 1997, A\&A, 317, 637

Capetti, A., Schreier, E. J., Axon, D., et al. 2000a, ApJ, 544, 269

Capetti, A., Trussoni, E., Celotti, A., Feretti, L., \& Chiaberge, M. 2000b, MNRAS, 318, 493

Capetti, A., Kleijn, G. V., \& Chiaberge, M. 2005, A\&A, 439, 935

Cardelli, J. A., Clayton, G. C., \& Mathis, J. S. 1989, ApJ, 345, 245

Chiaberge, M., Capetti, A., \& Celotti, A. 1999, A\&A, 349, 77

Chiaberge, M., Macchetto, F. D., Sparks, W. B., et al. 2002, ApJ, 571, 247

Cohen, M. H., Ogle, P. M., Tran, H. D., Goodrich, R. W., \& Miller, J. S. 1999, AJ, 118, 1963

Colla, G., Fanti, C., Fanti, R., et al. 1975, A\&AS, 20, 1

Crane, P., Peletier, R., Baxter, D., et al. 1993, ApJ, 402, L37

Evans, D. A., Worrall, D. M., Hardcastle, M. J., Kraft, R. P., \& Birkinshaw, M. 2006, ApJ, 642, 96

Fanaroff, B. L., \& Riley, J. M. 1974, MNRAS, 167, 31

Fossati, G., Maraschi, L., Celotti, A., Comastri, A., \& Ghisellini, G. 1998, MNRAS, 299, 433
Hardcastle, M. J., Alexander, P., Pooley, G. G., \& Riley, J. M. 1997, MNRAS, 288, L1

Gabuzda, D. C., Rastorgueva, E. A., Smith, P. S., \& O'Sullivan, S. P. 2006, MNRAS, 369, 1596

Hardcastle, M. J., \& Worrall, D. M. 2000, MNRAS, 314, 359

Hardcastle, M. J., Worrall, D. M., Birkinshaw, M., \& Canosa, C. M. 2003, MNRAS, 338, 176

Hough, J. H., Bailey, J. A., Rouse, M. F., \& Whittet, D. C. B. 1987, MNRAS, 227, 1P

Impey, C. D., \& Tapia, S. 1990, ApJ, 354, 124

Impey, C. D., Lawrence, C. R., \& Tapia, S. 1991, ApJ, 375, 46

Jannuzi, B. T., Smith, P. S., \& Elston, R. 1994, ApJ, 428, 130

Kharb, P., Shastri, P., \& Gabuzda, D. C. 2005, ApJ, 632, L69

Mahadevan, R. 1997, ApJ, 477, 585

Müller, S. A. H., Haas, M., Siebenmorgen, R., et al. 2004, A\&A, 426, L29

Narayan, R., \& Yi, I. 1995, ApJ, 444, 231

Ogle, P. M., Antonucci, R., \& Whysong, D. 2006 [arXiv:astro-ph/0612443]

Pellegrini, S. 2005, ApJ, 624, 155

Perlman, E. S., Biretta, J. A., Sparks, W. B., Macchetto, F. D., \& Leahy, J. P. 2001, ApJ, 551, 206

Perlman, E. S., Padgett, C. A., Georganopoulos, M., et al. 2006, ApJ, 651, 735

Schmidt, G. D., Elston, R., \& Lupie, O. L. 1992, AJ, 104, 1563

Schreier, E. J., Capetti, A., Macchetto, F., Sparks, W. B., \& Ford, H. J. 1996, ApJ, 459, 535

Serkowski, K., Mathewson, D. L., \& Ford, V. L. 1975, ApJ, 196, 261

Simmons, J. F. L., \& Stewart, B. G. 1985, A\&A, 142, 100

Smith, J. E., Young, S., Robinson, A., et al. 2002, MNRAS, 335, 773

Smith, J. E., Robinson, A., Alexander, D. M., et al. 2004, MNRAS, 350, 140

Stockman, H. S., Angel, J. R. P., \& Miley, G. K. 1979, ApJ, 227, L55

Trippe, S., Paumard, T., Ott, T., et al. 2006, MNRAS, accepted [arXiv:astro-ph/0611737]

Trussoni, E., Capetti, A., Celotti, A., Chiaberge, M., \& Feretti, L. 2003, A\&A, 403, 889

Ulvestad, J. S., \& Wilson, A. S. 1984, ApJ, 278, 544

Urry, C. M., \& Padovani, P. 1995, PASP, 107, 803

Venturi, T., Castaldini, C., Cotton, W. D., et al. 1995, ApJ, 454, 735

Wardle, J. F. C., \& Kronberg, P. P. 1974, ApJ, 194, 249

Whysong, D., \& Antonucci, R. 2004, ApJ, 602, 116

Xu, C., Baum, S. A., O’Dea, C. P., Wrobel, J. M., \& Condon, J. J. 2000, AJ, 120, 2950 